\title{
Butterflies (Lepidoptera Rhopalocera) of the Bor Wildlife Sanctuary, Wardha, Maharashtra, Central India
}

\author{
Ashish Tiple \\ Department of Zoology, Vidhyabharti College, Seloo, Wardha 442 104, India; e-mail: ashishdtiple@yahoo.co.in
}

\begin{abstract}
The diversity of butterfly species (Lepidoptera Rhopalocera) was studied in the Bor Wildlife Sanctuary, Wardha district area (Central India) of $138.12 \mathrm{~km}^{2}$ from 2011 to 2017. A total of 114 species of butterflies belonging to 6 families were recorded. Most of the butterflies recorded belong to the family Nymphalidae (35 species). 34 Lycaenidae species were recorded. A total of 18 Hesperiidae and 18 Pieridae species were recorded, 8 species were recorded from the Papilionidae and 1 species from the Riodinidae family. Among the 114 butterflies recorded, 9 species come under the protection category of the Indian Wild Life (protection) Act 1972 (i.e., Pachliopta hector, Appias albina, Appias libythea, Eurema andersonii, Euploea core, Hypolimnas misippus, Euchrysops cnejus, Lampides boeticus, Ionolyce helicon, Baoris farri). The observations support the value of the National Park (Reserve forest) area in providing valuable resources for butterflies.
\end{abstract}

KEY WORDS Lepidoptera; diversity; Bor wild life Sanctuary; Wardha; Mahrashtra.

Received 16.03.2018; accepted 22.05.2018; printed 30.09.2018; published online 05.10.2018

\section{INTRODUCTION}

Bor Wildlife Sanctuary was declared as a tiger reserve in July 2014. It is located near Hingani in Wardha District, Maharashtra. It is a home to a variety of wild animals. The reserve covers an area of $138.12 \mathrm{~km}^{2}$ (53.33 sq. mile) at $20^{\circ} 57^{\prime} \mathrm{N}$ and $78^{\circ} 37^{\prime} \mathrm{E}$ altitude, which includes the drainage basin of the Bor Dam. Bor Wildlife Sanctuary is covered with southern mixed dry deciduous forest. Teak, ain, tendu, and bamboo are the main species of flora in this sanctuary. Tigers, panthers, bisons, blue bulls, chitals, sambars, peacocks, barking deers, chinkara, monkeys, wild boars, bears, and wild dogs are the important faunas of the sanctuary. It represents the floral and faunal wealth of Satpuda-Maikal Landscape. Satpuda runs along the Northern Boundary of Maharashtra from West to
East and meets the Maikal Hill range which comes from Kanha (Figs. 1-3).

Among insect, butterflies are the most beautiful and colourful creatures on the earth, have a great aesthetic value and are called the flying jewels or winged jewels of nature. Butterflies are generally regarded as one of the best and most taxonomically studied groups of insects and well observed, not only by the lepidopterists and entomologists, but also by laymen. They are a very common and widespread species, but our understanding on their real biology and diversity may prove to be startlingly below common expectations (Willmott et al., 2001; Ackery, 1987; Tiple \& Khurad, 2009).

The butterflies are a very important unit of ecosystem due to the inter-relationship with plants diversity (Kunte, 2000). Their caterpillars can be reared at home and the transformation from cater- 
pillar to butterfly can easily be observed. Therefore, they make excellent subjects for natural history observations and scientific studies. Butterflies are very much important for the pollination as they tend tovisit different flowers for the nectar feeding, which make them an important unit of environment. Butterflies are very sensitive group to environment and are directly affected by changes in the habitats, atmospheric temperature, and weather conditions. They can be good indicators of environment changes (Tiple et al., 2006).

The Indian sub-region hosts about 1,504 species of butterflies, of which 351 in Peninsular India and 334 in the Western Ghats. In Central India, the butterfly diversity was reported earlier by Forsayeth (1884), Swinhoe (1886), Betham
(1890, 1891) and Witt (1909). D'Abreeu (1931) documented a total of 177 species occurring in the erstwhile Central Provinces (now Madhya Pradesh and Vidarbha). In the recent past, several workers have studied butterflies from urban, rural, and protected areas of Vidarbha. 65 species belonging to 52 genera representing 7 families from Pench Tiger Reserve (Maharashtra) (Sharma \& Radhakrishnan, 2005), 68 species of butterflies of 50 genera were recorded from Tadoba Andhari Tiger Reserve (Sharma \& Radhakrishnan, 2006) and 103 species of butterflies were recorded from Melghat Tiger Reserve (Wadatkar, 2008). Tiple \& Khurad (2009) reported 145 species of butterflies, of which 62 species were new records for Nagpur city. Recently, Tiple (2010) documented 111 species of

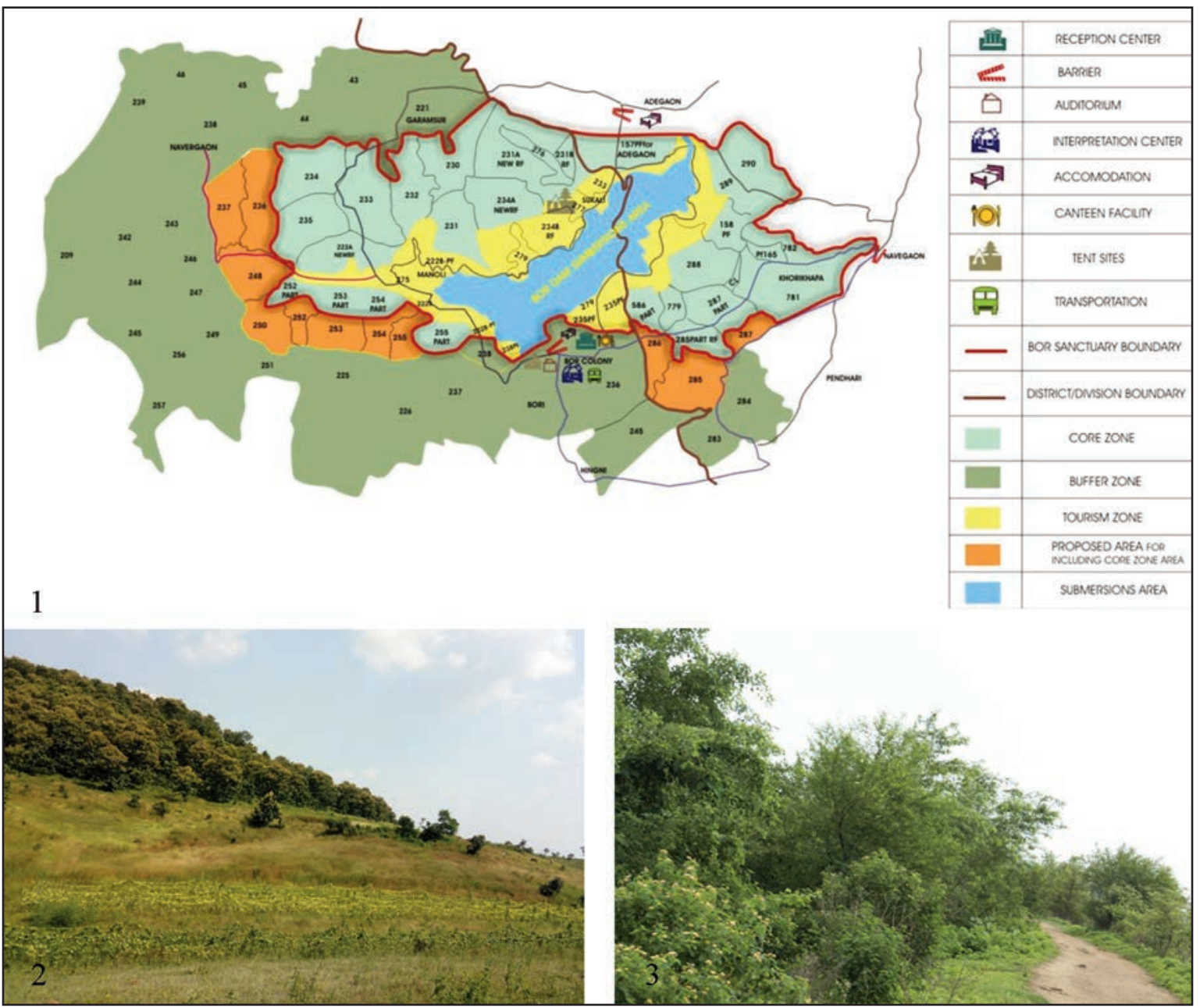

Figure 1. Location map of Bor Wild Life Sanctuary in district Wardha, Maharashtra, Central India (Image Source www.wikimapia.org ). Figures 2, 3. Natural environment of Bor Wildlife Santury. 
butterflies in Tadoba National Park. The present study was started to examine the diversity of butterflies from Bor Wildlife Sanctuary, Wardha, since there was no known published checklist of butterflies in the Bor Wildlife Sanctuary.

\section{MATERIAL AND METHODS}

Butterfly were surveyed in different regions of the Bor wildlife Sanctuary since 2011 to 2017 along the reserve forest areas, buffer zone, lakes, rivers, and surrounding areas. Identification of the butterflies was primarily made directly in the field. In critical condition specimens were collected only with handheld aerial sweep nets and subsequently released without harm. Butterflies were identified from Wynter-Blyth (1957) and Kunte (2000). All scientific names follow Varshney (1983) and common English names are after Wynter-Blyth (1957). Based on the number of sightings, the butterfly species were categorized into very rare $(<2$ sightings), rare (2-15 sightings), not rare (15-50 sightings), common (50-100 sightings) and very common (more than 100 sightings).

\section{RESULTS AND DISCUSSION}

During the course of study, 114 species of butterflies belonging to 6 families were recorded (Tables 1-3, Figs. 4-15). Most of the butterflies recorded belong to the Nymphalidae ( 35 species) and Lycaenidae (34 species), followed by Pieridae (18 species), Hesperiidae (18 species), Papilionidae ( 8 species), and 1 from the Riodinidae (see Table 1). Among the 114 butterflies recorded, 9 species come under the protection category of the Indian Wildlife (protection) Act 1972 (Tiple, 2011; Gupta $\&$ Mondal, 2005) (i.e., Pachliopta hector, Appias albina, A. libythea, Eurema andersonii, Euploea core, Hypolimnas misippus, Euchrysops cnejus, Lampides boeticus, Ionolyce helicon, Baoris farri).

Seasonal patterns of species richness (flight periods) in Bor Wildlife Sanctuary, are presented in figure 16. Most butterfly species were observed from the monsoon (hot/wet season) to early winter (cool/wet season), but thereafter declined in early summer (March). Among the 114 species of butterflies (Papilio demoleus, Cepora nerissa, Eurema brigitta, E. hecabe, Danaus chrysippus, Euploea core, Hypolimnas misippus, Junonia lemonias, Melanitis leda, Tirumala limniace, Castalius rosimon, Catochrysops strabo, Luthrodes pandava, Zizeeria karsandra, Borbo cinnara) occurred throughout the year (January-December), whereas the remaining 99 species of butterflies were prominently observed only after June-July up to the beginning of summer (April-May). Increasing species abundance from the beginning of the monsoon (June-July) until the early winter (August-November) and decline in species abundance from late winter (January - February) up to the end of summer have also been reported by Tiple et al. (2007), Tiple $\&$ Khurad (2009), and Tiple $(2010,2011)$ in similar climatic conditions in this region of Central India. They further demonstrated that most of the species were noticeably absent in the disturbed and human impacted sites (gardens, plantation, and grassland) and there was no occurrence of unique species in moderately disturbed areas comparable to those of less disturbed wild areas. The present study area, the Bor Wildlife Sanctuary and surrounding areas, is always disturbed and impacted by humans, which may be the reason for overall reduction of the uniqueness of the species from disturbed and impacted sites as compared to the other sites.

In the present study, seasonal occurrence of butterfly species was high from monsoon (hot/wet season) to early winter (cool/wet season), but thereafter declined in early summer (March). The cause of this decline might be non-availability of nectar and larval host plants and scarcity of water (Tiple \& Khu$\mathrm{rad}, 2009)$. Of course, this is but one aspect of the resources used by butterflies in the reserve forest area and a complete picture of habitat structure can only be obtained by researching into all consumable and utility resources (Dennis et al., 2003). Butterfly populations would clearly benefit from planting indigenous, as opposed to exotic, nectar and larval host plants which are the sources of various proteins and salts that are essential for the buildup of a healthy and genetically diverse butterfly population (Tiple et al., 2006). In particular, attention should be paid to the seasonal availability of resources and to resources for less common butterflies on this reserve forest area. All in all, this reserve forest area (Bor Wildlife Sanctuary) provides rich ground not just for conservation, but also for research into butterfly biology for students. 


\begin{tabular}{|c|c|c|c|c|}
\hline $\begin{array}{l}\text { Sr. } \\
\text { No. }\end{array}$ & Scientific Name & Common Name & Status & $\begin{array}{l}\text { Occurrence } \\
\text { (months) }\end{array}$ \\
\hline & Papilionidae(08) & & & \\
\hline 1 & Graphium agamemnon (Linnaeus, 1758) & Tailed Jay & $\mathrm{C}$ & $8-1$ \\
\hline 2 & Graphium doson (C. et R. Felder, 1864) & Common Jay & $\mathrm{C}$ & $8-1$ \\
\hline 3 & Graphium nomius (Esper, 1799) & Spot Swordtail & $\mathrm{R}$ & $3-7$ \\
\hline 4 & Pachliopta aristolochiae (Fabricius, 1775) & Common Rose & $\mathrm{C}$ & $7-2$ \\
\hline 5 & Pachliopta hector (Linnaeus, 1758) & Crimson Rose & $\mathrm{C}$ & $7-2$ \\
\hline 6 & Papilio demoleus Linnaeus, 1758 & Lime Butterfly & VC & $1-12$ \\
\hline 7 & Papilio polymnestor Cramer, [1775] & Blue Mormon & $\mathrm{R}$ & $9-11$ \\
\hline \multirow[t]{2}{*}{8} & Papilio polytes Linnaeus, 1758 & Common Mormon & $\mathrm{VC}$ & $7-2$ \\
\hline & Pieridae (18) & & & \\
\hline 9 & Belenois aurota (Fabricius, 1793) & Pioneer & VC & $9-2$ \\
\hline 10 & Appias albina (Boisduval, 1836) & Common Albatross & VR & $11-12$ \\
\hline 11 & Catopsilia pomona (Fabricius, 1775) & Common or Lemon Emigrant & VC & $1-12$ \\
\hline 12 & Catopsilia pyranthe (Linnaeus, 1758) & Mottled Emigrant & VC & $7-2$ \\
\hline 13 & Cepora nerissa (Fabricius, 1775) & Common Gull & $\mathrm{VC}$ & $1-12$ \\
\hline 14 & Colotis fausta (Olivier, 1804) & Large Salmon Arab & $\mathrm{C}$ & $8-11$ \\
\hline 15 & Colotis danae (Fabricius, 1775) & Crimson Tip & $\mathrm{VC}$ & $6-10$ \\
\hline 16 & Colotis etrida (Boisduval, 1836) & Small Orange Tip & $\mathrm{C}$ & $8-12$ \\
\hline 17 & Colotis aurora Cramer, 1780 & Plain Orange Tip & $\mathrm{R}$ & 8 \\
\hline 18 & Ixias pyrene Linnaeus, 1764 & Yellow Orange Tip & $\mathrm{R}$ & $8-9$ \\
\hline 19 & Delias eucharis (Drury, 1773) & Common Jezebel & VC & $7-2$ \\
\hline 20 & Eurema blanda (Boisduval, 1836) & Three-Spot Grass Yellow & $\mathrm{C}$ & $7-2$ \\
\hline 21 & Eurema brigitta (Stoll, [1780]) & Small Grass Yellow & $\mathrm{C}$ & $1-12$ \\
\hline 22 & Eurema hecabe (Linnaeus, 1758) & Common Grass Yellow & VC & $1-12$ \\
\hline 23 & Eurema laeta (Boisduval, 1836) & Spotless Grass Yellow & $\mathrm{VC}$ & $7-12$ \\
\hline 24 & Ixias marianne (Cramer, [1779]) & White Orange Tip & $\mathrm{C}$ & $8-11$ \\
\hline 25 & Leptosia nina (Fabricius, 1793) & Psyche & $\mathrm{C}$ & $8-1$ \\
\hline \multirow[t]{2}{*}{26} & Pareronia hippie (Cramer, [1776]) & Common Wanderer & $\mathrm{C}$ & $8-2$ \\
\hline & Nymphalidae (35) & & & \\
\hline 27 & Acraea violae (Fabricius, 1793) & Tawny Coster & VC & $10-12$ \\
\hline 28 & Ariadne ariadne (Linnaeus, 1763) & Angled Castor & VC & $9-2$ \\
\hline 29 & Ariadne merione (Cramer, [1777]) & Common Castor & $\mathrm{C}$ & $10-2$ \\
\hline 30 & Byblia ilithyia (Drury, [1773]) & Joker & $\mathrm{C}$ & $6-12$ \\
\hline 31 & Charaxes solon (Fabricius, 1793) & Black Rajah & $\mathrm{C}$ & $10-2$ \\
\hline 32 & Vanessa cardui (Linnaeus, 1758) & Painted Lady & $\mathrm{C}$ & $6-8$ \\
\hline 33 & Danaus chrysippus (Linnaeus, 1758) & Plain Tiger & $\mathrm{VC}$ & $1-12$ \\
\hline 34 & Danaus genutia (Cramer, [1779]) & Striped Tiger & VC & $10-6$ \\
\hline 35 & Euploea core (Cramer, [1780]) & Common Indian Crow & VC & $1-12$ \\
\hline 36 & Euthalia aconthea (Cramer, [1777]) & Common Baron & $\mathrm{C}$ & $8-11$ \\
\hline
\end{tabular}

Table 1/1. List of Lepidoptera Rhopalocera recorded from Bor Wild Life Sanctuary, Wardha, Maharashtra, Central India together with common name, status, and Occurrence. 


\begin{tabular}{|c|c|c|c|c|}
\hline 37 & Hypolimnas bolina (Linnaeus, 1758) & Great Eggfly & $\mathrm{C}$ & $6-3$ \\
\hline 38 & Hypolimnas misippus (Linnaeus, 1764) & Danaid Eggfly & $\mathrm{C}$ & $1-12$ \\
\hline 39 & Junonia almana (Linnaeus, 1758) & Peacock Pansy & VC & $6-2$ \\
\hline 40 & Junonia atlites (Linnaeus, 1763) & Grey Pansy & $\mathrm{VC}$ & $7-2$ \\
\hline 41 & Junonia hierta (Fabricius, 1798) & Yellow Pansy & $\mathrm{C}$ & $8-2$ \\
\hline 42 & Junonia iphita (Cramer, [1779]) & Chocolate Pansy & VC & $8-3$ \\
\hline 43 & Junonia lemonias (Linnaeus, 1758) & Lemon Pansy & $\mathrm{VC}$ & $1-12$ \\
\hline 44 & Junonia orithya (Linnaeus, 1758) & Blue Pansy & VC & $10-4$ \\
\hline 45 & Lethe europa (Fabricius, 1775) & Bamboo Treebrown & $\mathrm{C}$ & $8-3$ \\
\hline 46 & Melanitis leda (Linnaeus, 1758) & Common Evening Brown & VC & $1-12$ \\
\hline 47 & Moduza procris (Cramer, [1777]) & Commander & $\mathrm{C}$ & $8-1$ \\
\hline 48 & Mycalesis intermedia (Moore, [1892]) & Intermediate Bushbrown & $\mathrm{R}$ & $8-1$ \\
\hline 49 & Mycalesis mineus (Linnaeus, 1758) & Dark Branded Bushbrown & $\mathrm{C}$ & $8-3$ \\
\hline 50 & Mycalesis perseus (Fabricius, 1775) & Common Bushbrown & $\mathrm{VC}$ & $7-3$ \\
\hline 51 & Phaedyma columella (Cramer, $[1780])$ & Short-banded Sailer & $\mathrm{C}$ & $9-11$ \\
\hline 52 & Neptis hylas (Linnaeus, 1758 ) & Common Sailer & VC & $7-3$ \\
\hline 53 & Neptis jumbah Moore, [1858] & Chestnut-Streaked Sailer & $\mathrm{C}$ & $9-11$ \\
\hline 54 & Phalanta phalantha (Drury, [1773]) & Common Leopard & VC & $6-3$ \\
\hline 55 & Charaxes agrarius (Swinhoe, 1887) & Anomalous Nawab & $\mathrm{C}$ & $2-3$ \\
\hline 56 & Symphaedra nais (Forster, 1771) & Baronet & $\mathrm{C}$ & $10-3$ \\
\hline 57 & Tirumala limniace (Cramer, [1775]) & Blue Tiger & $\mathrm{VC}$ & $1-12$ \\
\hline 58 & Ypthima asterope (Klug, 1832) & Common Threering & VC & $7-9$ \\
\hline 59 & Ypthima baldus (Fabricius, 1775) & Common Fivering & $\mathrm{C}$ & $9-10$ \\
\hline 60 & Ypthima huebneri (Kirby, 1871) & Common Fourring & $\mathrm{C}$ & $11-12$ \\
\hline \multirow[t]{2}{*}{61} & Ypthima inica (Hewitson, 1865) & Lesser Threering & $\mathrm{C}$ & $9-12$ \\
\hline & Riodinidae (1) & & & \\
\hline \multirow[t]{2}{*}{62} & Abisara bifasciata Moore, 1877 & Double-banded Judy & $\mathrm{R}$ & $8-10$ \\
\hline & Lycaenidae (34) & & & \\
\hline 63 & Acytolepis puspa (Horsfield, [1828]) & Common Hedge Blue & VC & $9-2$ \\
\hline 64 & Amblypodia anita Hewitson, 1862 & Leaf Blue & VC & $8-9$ \\
\hline 65 & Anthene lycaenina (Felder, 1868) & Pointed Ciliate Blue & $\mathrm{C}$ & $8-11$ \\
\hline 66 & Arhopala amantes (Hewitson, 1862) & Large Oakblue & $\mathrm{C}$ & $5-6$ \\
\hline 67 & $\begin{array}{l}\text { Arhopala pseudocentaurus (Doubleday, } \\
\text { 1847) }\end{array}$ & Western Centaur Oakblue & VR & 10 \\
\hline 68 & Azamus jesous (Guérin-Méneville, 1849) & African Babul Blue & $\mathrm{C}$ & $10-2$ \\
\hline 69 & Azanus ubaldus (Stoll, [1782]) & Bright Babul Blue & $\mathrm{R}$ & $11-2$ \\
\hline 70 & Castalius rosimon (Fabricius, 1775) & Common Pierrot & VC & $1-12$ \\
\hline 71 & Catochrysops strabo (Fabricius, 1793) & Forget-Me-Not & VC & $1-12$ \\
\hline 72 & Chilades lajus (Stoll, [1780]) & Lime Blue & VC & $8-12$ \\
\hline 73 & Luthrodes pandava (Horsfield, [1829]) & Plains Cupid & VC & $1-12$ \\
\hline 74 & Chilades parrhasius (Fabricius, 1793) & Small Cupid & $\mathrm{C}$ & $7-2$ \\
\hline 75 & Freyeria putli (Kollar, [1844]) & Eastern Grass Jewel & $\mathrm{C}$ & $7-12$ \\
\hline
\end{tabular}

Table 1/2. List of Lepidoptera Rhopalocera recorded from Bor Wild Life Sanctuary, Wardha, Maharashtra, Central India together with common name, status, and Occurrence. 


\begin{tabular}{|c|c|c|c|c|}
\hline 76 & Virachola isocrates (Fabricius, 1793) & Common Guava Blue & $\mathrm{C}$ & $8-10$ \\
\hline 77 & Euchrysops cnejus (Fabricius, 1798) & Gram Blue & VC & $6-3$ \\
\hline 78 & Everes lacturnus (Godart, [1824]) & Indian Cupid & $\mathrm{C}$ & $12-2$ \\
\hline 79 & Jamides bochus (Stoll, [1782]) & Dark Cerulean & $\mathrm{C}$ & $7-2$ \\
\hline 80 & Jamides celeno (Cramer, [1775]) & Common Cerulean & VC & $7-3$ \\
\hline 81 & Lampides boeticus (Linnaeus, 1767) & Pea Blue & VC & $8-3$ \\
\hline 82 & Leptotes plinius (Fabricius, 1793) & Zebra Blue & $\mathrm{VC}$ & $7-3$ \\
\hline 83 & Petrelaea dana (de Nicéville, [1884]) & Dingy Lineblue & $\mathrm{C}$ & $8-9$ \\
\hline 84 & Prosotas dubiosa (Semper, [1879]) & Tailless Lineblue & $\mathrm{C}$ & $7-9$ \\
\hline 85 & Prosotas nora (Felder, 1860) & Common Lineblue & $\mathrm{C}$ & $7-3$ \\
\hline 86 & Psuedozizeeria maha (Kollar, [1844]) & Pale Grass Blue & $\mathrm{C}$ & $8-3$ \\
\hline 87 & Rapala iarbus (Fabricius, 1787) & Common Red Flash & $\mathrm{C}$ & $8-12$ \\
\hline 88 & Spindasis ictis (Hewitson, 1865) & Common Shot Silverline & $\mathrm{C}$ & $6-8$ \\
\hline 89 & Spindasis schistacea (Moore, [1881]) & Plumbeous Silverline & $\mathrm{C}$ & $7-8$ \\
\hline 90 & Spindasis vulcanus (Fabricius, 1775) & Common Silverline & VC & $8-2$ \\
\hline 91 & $\begin{array}{l}\text { Tarucus balkanicus nigra } \text { Bethune-Baker, } \\
\text { [1918] }\end{array}$ & Black-spotted Pierrot & $\mathrm{C}$ & $8-1$ \\
\hline 92 & Tarucus callinara Butler, 1886 & Spotted Pierrot & $\mathrm{C}$ & $8-2$ \\
\hline 93 & Tarucus nara (Kollar, 1848) & $\begin{array}{l}\text { Rounded Pierrot/ Striped } \\
\text { Pierrot }\end{array}$ & $\mathrm{VC}$ & $7-2$ \\
\hline 94 & Zizeeria karsandra (Moore, 1865) & Dark Grass Blue & VC & $1-12$ \\
\hline 95 & Zizina otis (Fabricius, 1787) & Lesser Grass Blue & $\mathrm{VC}$ & $6-3$ \\
\hline \multirow[t]{2}{*}{96} & Zizula hylax (Fabricius, 1775) & Tiny Grass Blue & $\mathrm{VC}$ & $6-3$ \\
\hline & Hesperiidae (18) & & & \\
\hline 97 & Badamia exclamationis (Fabricius, 1775) & Brown Awl & $\mathrm{VC}$ & $8-11$ \\
\hline 98 & Baoris farri (Moore, 1878) & Paintbrush Swift & $\mathrm{R}$ & $10-12$ \\
\hline 99 & Borbo bevani (Moore, 1878) & Bevan's Swift & $\mathrm{C}$ & $8-11$ \\
\hline 100 & Borbo cinnara (Wallace, 1866) & Rice Swift & VC & $1-12$ \\
\hline 101 & Caltoris kumara (Moore, 1878) & Blank Swift & $\mathrm{C}$ & $10-12$ \\
\hline 102 & Celaenorrhinus leucocera (Kollar, [1844]) & Common Spotted Flat & $\mathrm{R}$ & $8-9$ \\
\hline 103 & Coladenia indrani (Moore, $[1866])$ & Tricolour Pied Flat & $\mathrm{C}$ & $9-12$ \\
\hline 104 & Hasora badra (Moore, [1858]) & Common Awl & $\mathrm{C}$ & $7-9$ \\
\hline 105 & Hasora chromus (Cramer, [1780]) & Common Banded Awl & $\mathrm{VC}$ & $9-10$ \\
\hline 106 & Hasora taminatus (Hübner, 1818) & White Banded Awl & $\mathrm{C}$ & $8-10$ \\
\hline 107 & Parnara naso(Fabricius, 1798) & Straight Swift & $\mathrm{C}$ & $9-12$ \\
\hline 108 & Pelopidas mathias (Fabricius, 1798) & Small Branded Swift & $\mathrm{VC}$ & $7-12$ \\
\hline 109 & Pseudocoladenia dan (Fabricius, 1787) & Fulvous Pied Flat & $\mathrm{R}$ & 9 \\
\hline 110 & Spialia galba (Fabricius, 1793) & Indian Skipper & VC & $8-3$ \\
\hline 111 & Suastus gremius (Fabricius, 1798) & Indian Palm Bob & $\mathrm{C}$ & $7-12$ \\
\hline 112 & Telicota bambusae (Moore, 1878) & Dark Palm Dart & $\mathrm{VC}$ & $8-12$ \\
\hline 113 & Telicota colon (Fabricius, 1775) & Pale Palm Dart & $\mathrm{C}$ & $7-10$ \\
\hline 114 & Udaspes folus (Cramer, [1775]) & Grass Demon & $\mathrm{C}$ & $9-12$ \\
\hline
\end{tabular}

Table 1/3. List of Lepidoptera Rhopalocera recorded from Bor Wild Life Sanctuary, Wardha, Maharashtra, Central India together with common name, status, and Occurrence. 


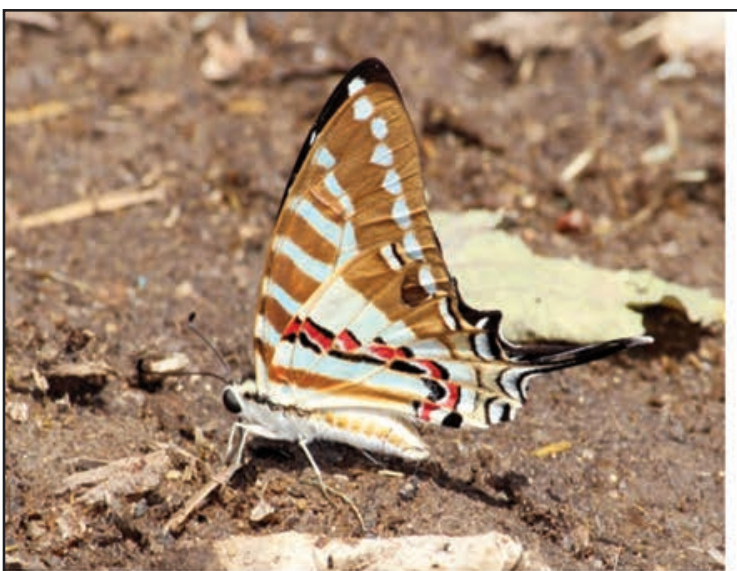

4

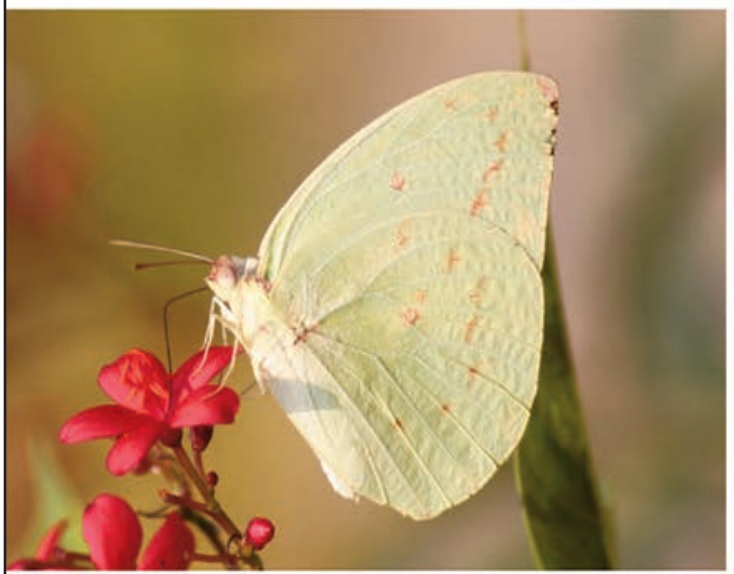

6

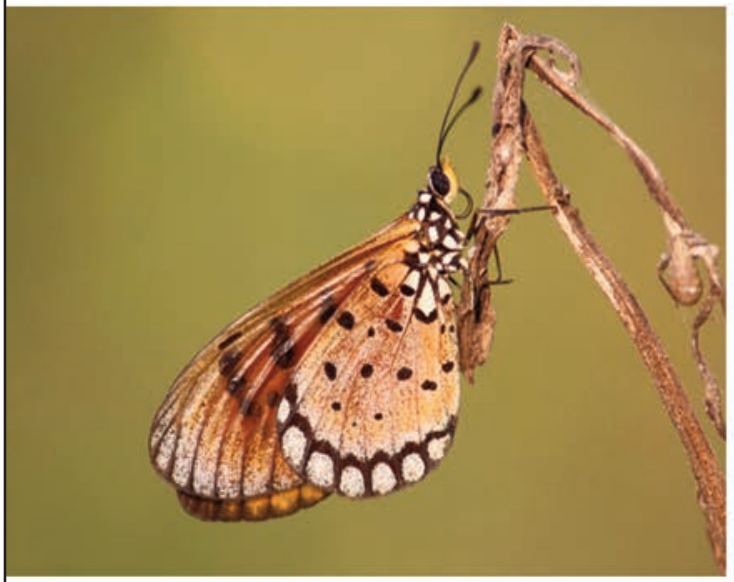

8

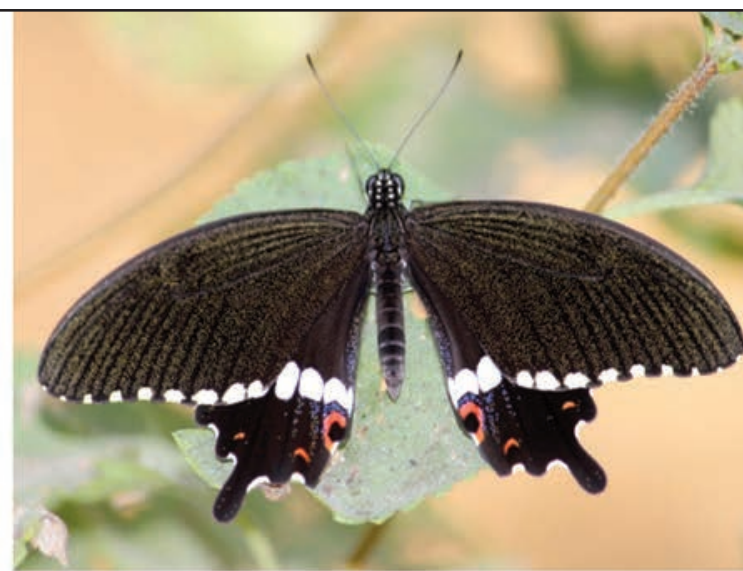

5

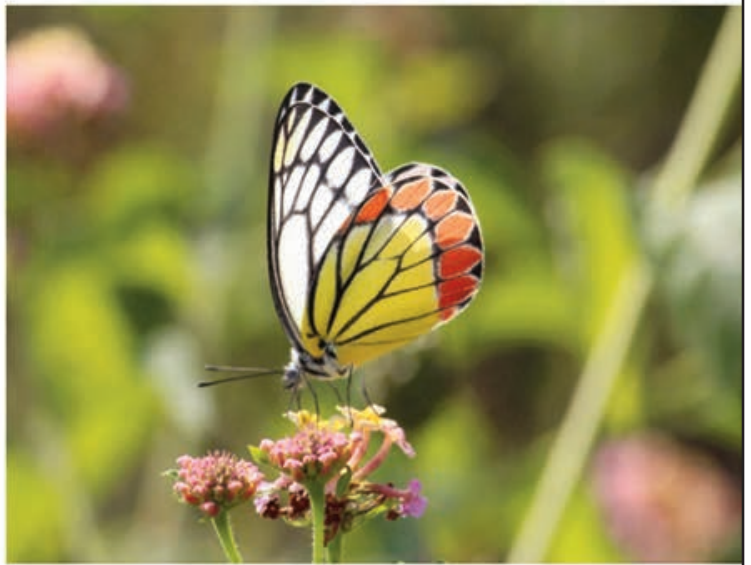

7

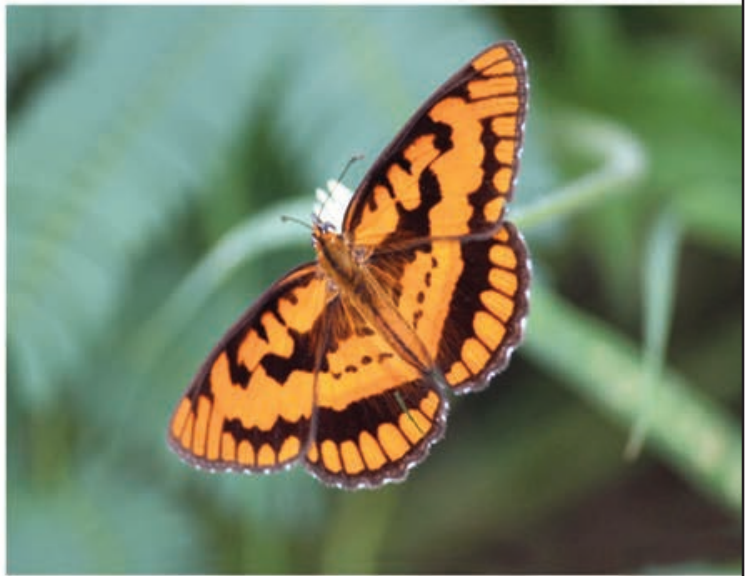

9

Figures 4-9. Lepidoptera Rhopalocera recorded from the natural environment of Bor Wildlife Santury, Wardha, Maharashtra, Central India. Figure 4: Spot Swordtail, Graphium nomius (Esper, 1799). Figure 5: Common Mormon, Papilio polytes Linnaeus, 1758. Figure 6: Common Emigrant, Catopsilia pomona (Fabricius, 1775). Figure 7: Common Jezebel, Delias eucharis (Drury, 1773). Figure 8: Tawny Coster, Acraea violae (Fabricius, 1793). Figure 9: Joker, Byblia ilithyia (Drury, [1773]). 


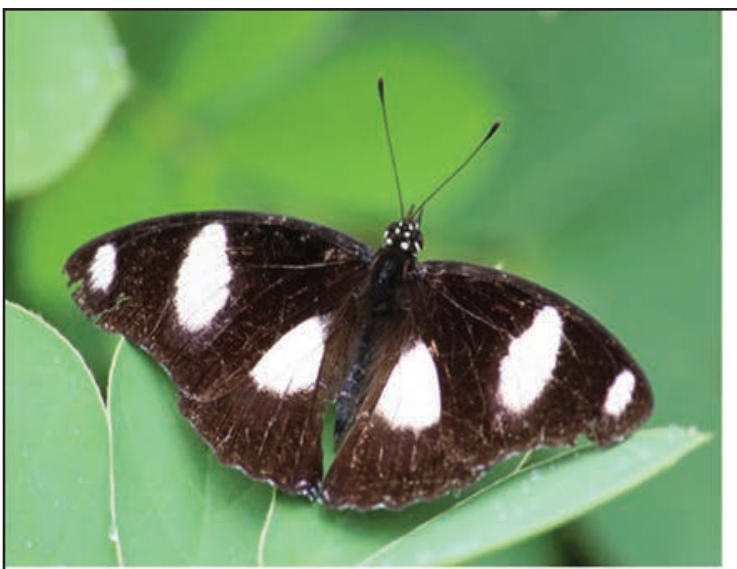

10

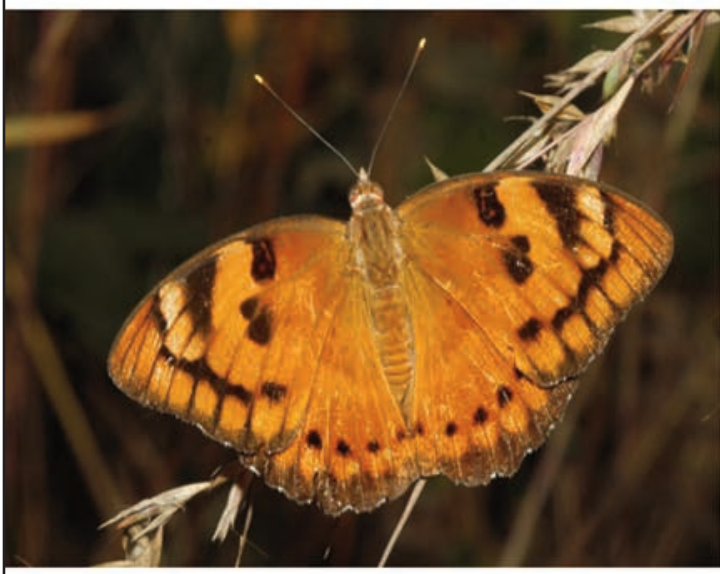

12

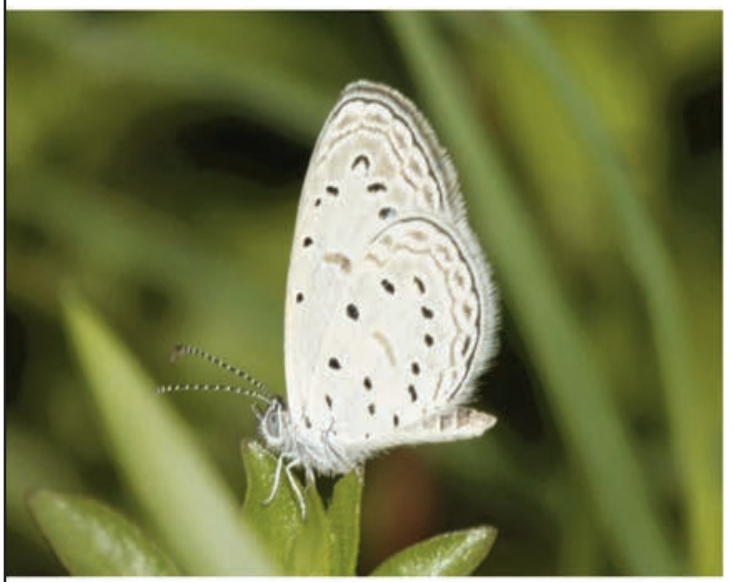

14

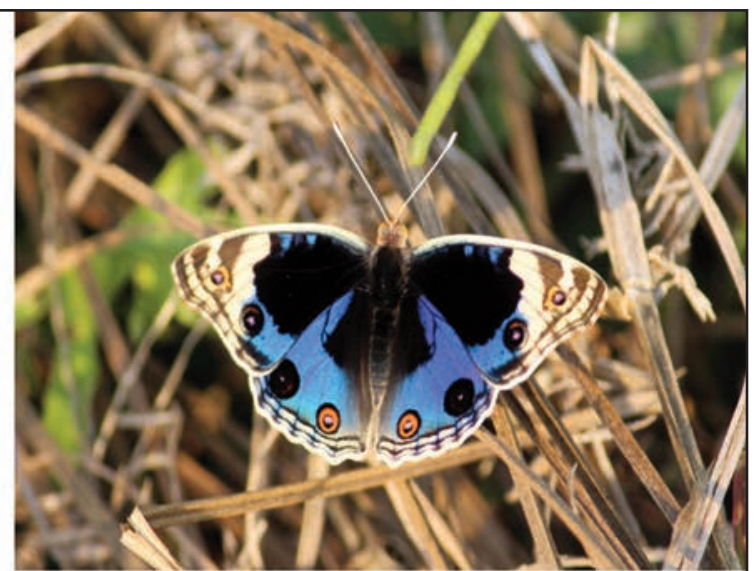

11

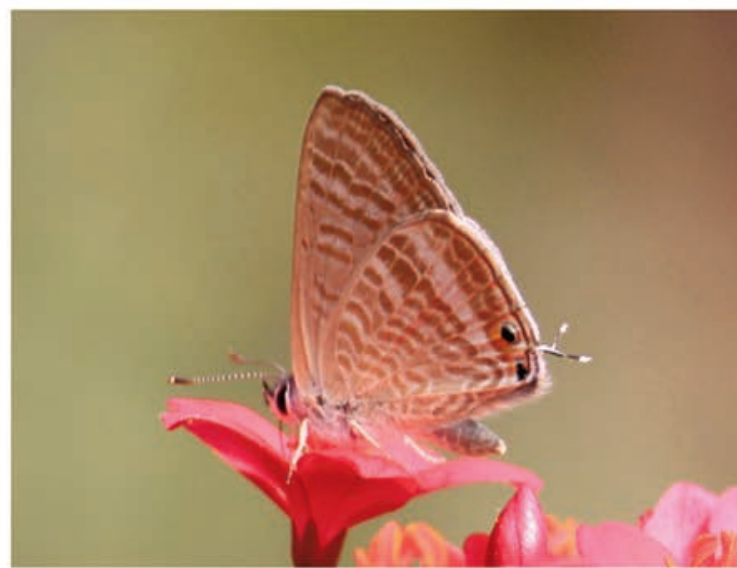

13

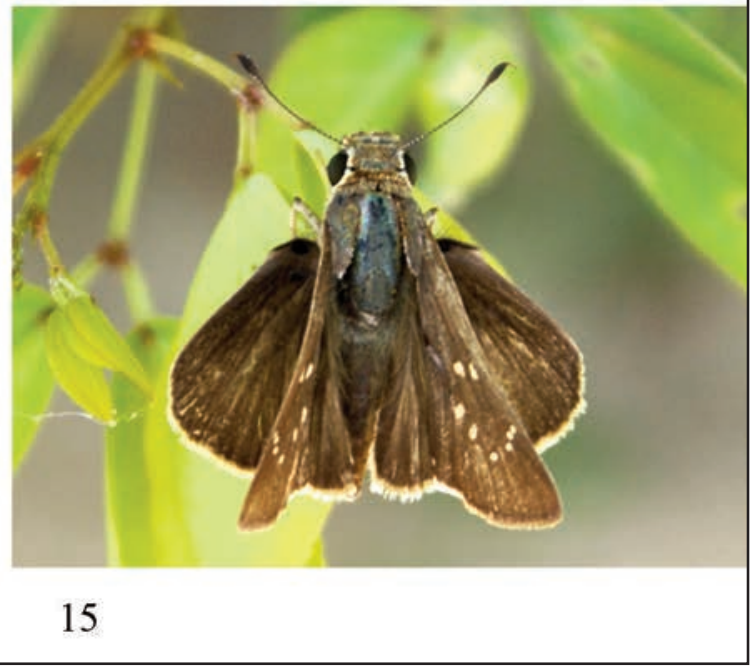

Figures 10-15. Lepidoptera Rhopalocera recorded from the natural environment of Bor Wildlife Santury, Wardha, Maharashtra, Central India. Figure 10: Danaid Eggfly, Hypolimnas misippus (Linnaeus, 1764). Figure 11: Blue Pansy, Junonia orithya (Linnaeus, 1758). Figure 12: Baronet, Symphaedra nais (Forster, 1771). Figure 13: Pea Blue, Lampides boeticus (Linnaeus, 1767). Figure 14: Tiny Grass Blue, Zizula hylax (Fabricius, 1775). Figure 15: Small Branded Swift, Pelopidas mathias (Fabricius, 1798). 


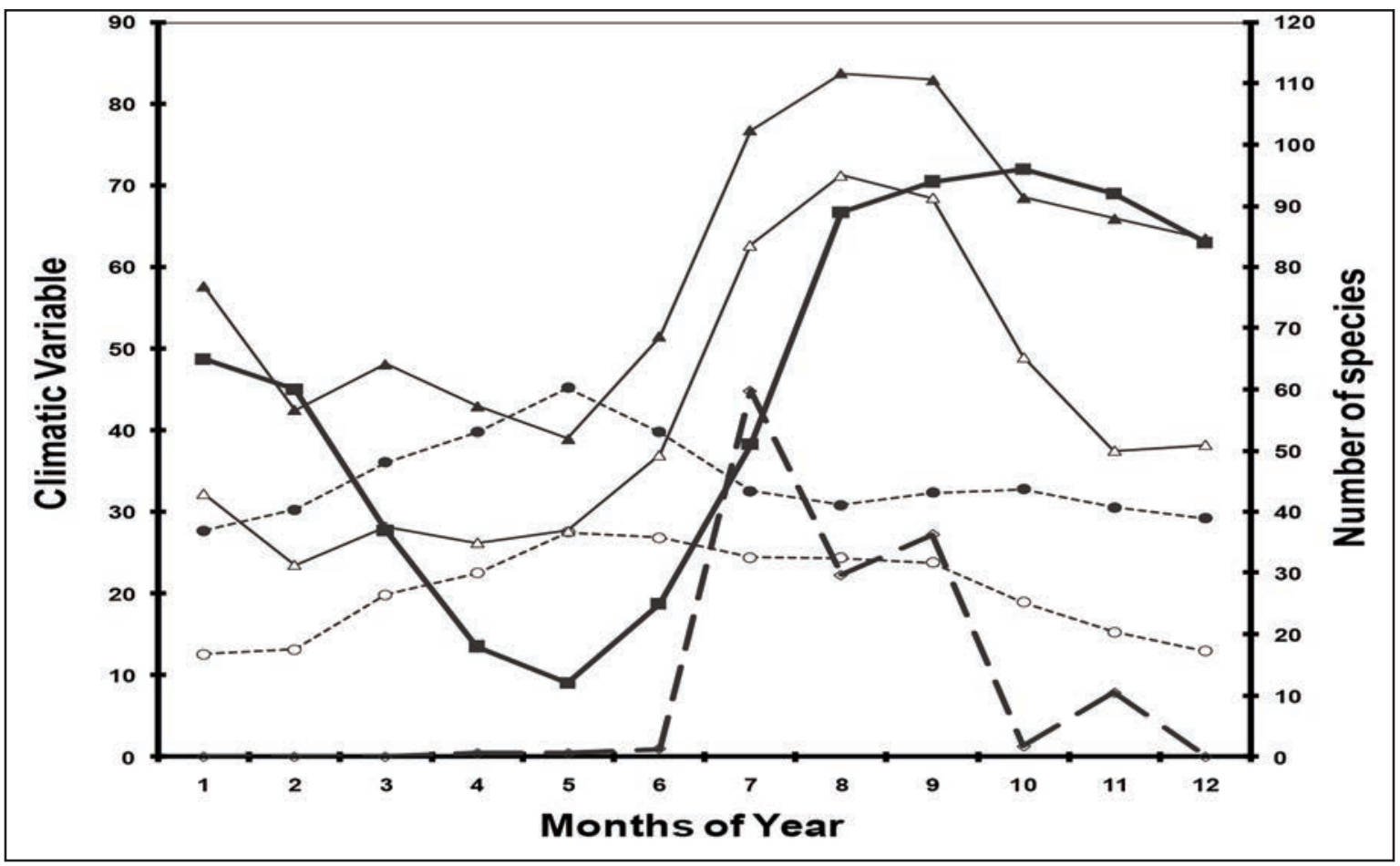

Figure 16. Seasonal distribution in number of species inside and around Bor Wild Life Sanctuary. Right scale: black squares, number of species. Left scale: climate variables, circles, temperatures $\left(0^{\circ} \mathrm{C}\right)$; closed circles, maximum mean temperatures $\left(0{ }^{\circ} \mathrm{C}\right)$; open, minimum mean temperatures $\left(0{ }^{\circ} \mathrm{C}\right)$; triangles, relative humidity $(\%)$; closed triangles, maximum relative humidity $(\%)$; open triangles, minimum relative humidity $(\%)$; diamonds, rainfall $(\mathrm{cm})$.

\section{REFERENCES}

Ackery P.R., 1987. Diversity and phantom competition in African acraeine butterflies. Biological Journal of the Linnean Society, 30: 291-297.

Betham J.A., 1890. The butterflies of the Central Provinces. Journal of the Bombay Natural History Society, 5: 19-28; 151-161; 279-286.

Betham J.A., 1891. The butterflies of the Central Provinces. Journal of the Bombay Natural History Society, 6: 175-183; 318-331.

D’Abreeu E.A., 1931. The central provinces butterfly list. Records of the Nagpur museum number VII. Government printing city press, $39 \mathrm{pp}$.

Dennis R.L.H., Shreeve T.G. \& Van Dyck H., 2003. Towards a resource-based concept for habitat: a butterfly biology viewpoint. Oikos, 102: 417-426.

Forsayeth R.W., 1884. Life history of sixty species of Lepidoptera observed in Mhow, Central India. Transactions of the Entomological Society of London, 3: $377-419$

Gupta I.J. \& Mondal D.K., 2005. Red Data Book, Part II: Butterflies of India. Zoological Society of India, Kolkata, 1-535.
Kunte K., 2000. Butterflies of Peninsular India. Universities Press (Hyderabad) and Indian Academy of Sciences (Bangalore), $254 \mathrm{pp}$.

Sharma R.M. \& Radhakrishnan C., 2005. Insecta: Lepidoptera (Rhopalocera and Grypocera). Fauna of Pench National Park, Conservation area series. Zoological Survey of India, 20: 251-274.

Sharma R.M. \& Radhakrishnan C., 2006. Insecta: Lepidoptera (Rhopalocera and Grypocera). Fauna of Todoba Andhari Tiger Reserve, Conservation area series. Zoological Survey of India, 25: 255-277.

Swinhoe C., 1886. On the Lepidoptera of Mhow. Proceedings of Zoological Society of London, 421-465.

Tiple A.D., 2010. Butterfly Fauna of Tadoba National Park and Surroundings, Chandrapur, Maharashtra (Central India). Hislopia, 3: 1-9.

Tiple A.D., 2011. Butterflies of Vidarbha region, Maharashtra State, central India. Journal of Threatened Taxa, 3: 1469-1477.

Tiple A.D. \& Khurad A.M., 2009. Butterfly Species Diversity, Habitats and Seasonal Distribution in and around Nagpur City, Central India. World Journal of Zoology, 4: 153-162.

Tiple A.D., Deshmukh V.P. \& Dennis R.L.H., 2006. Factors influencing nectar plant resource visits by but- 
terflies on a university campus: implications for conservation. Nota Lepidopterologica, 28: 213-224.

Tiple A.D., Khurad A.M. \& Dennis R.L.H., 2007. Butterfly diversity in relation to a human-impact gradient on an Indian university campus. Nota Lepidopterologica, 30: 179-188.

Varshney R.K.,1983. Index Rhopalocera indica part II. Common names of Butterflies from India and neighbouring countries. Records of the Zoological Survey of India, Occasional Paper no. 47: 1-49

Wadatkar J.S., 2008. Butterflies of Melghat Tiger Reserve, Maharashtra with notes on their abundance, status. Mel-Vyaghra, 8: 1-5.

Willmott K.R., Hall J.P.W. \& Lamas G., 2001. Systematics of Hypanartia (Lepidoptera: Nymphalidae: Nymphalinae), with a test for geographical speciation mechanisms in the Andes. Systematic Entomology, 26: 369-399.

Witt D.O., 1909. The butterflies (Rhopalocera) of the Nimar district, Central Provinces. Journal of the Bombay Natural History Society, 19: 564-571.

Wynter-Blyth M.A., 1957. Butterflies of the Indian Region. Published by Bombay Natural History Society, Bombay, 523 pp. 